TITLE:

\title{
Analysis of the filtered-X LMS algorithm and a related new algorithm for active control of multitonal noise
}

\author{
$\operatorname{AUTHOR}(\mathrm{S})$ :
}

Hinamoto, Y; Sakai, H

\section{CITATION:}

Hinamoto, Y...[et al]. Analysis of the filtered-X LMS algorithm and a related new algorithm for active control of multitonal noise. IEEE TRANSACTIONS ON AUDIO SPEECH AND LANGUAGE PROCESSING 2006, 14(1): 123-130

\section{ISSUE DATE:}

2006-01

URL:

http://hdl.handle.net/2433/50543

\section{RIGHT:}

(c)2006 IEEE. Personal use of this material is permitted. However, permission to reprint/republish this material for advertising or promotional purposes or for creating new collective works for resale or redistribution to servers or lists, or to reuse any copyrighted component of this work in other works must be obtained from the IEEE. 


\title{
Analysis of the Filtered-X LMS Algorithm and a Related New Algorithm for Active Control of Multitonal Noise
}

\author{
Yoichi Hinamoto, Student Member, IEEE, and Hideaki Sakai, Senior Member, IEEE
}

\begin{abstract}
In the presence of tonal noise generated by periodic noise source like rotating machines, the filtered-X LMS (FXLMS) algorithm is used for active control of such noises. However, the algorithm is derived under the assumption of slow adaptation limit and the exact analysis of the algorithm is restricted to the case of one real sinusoid in the literature. In this paper, for the general case of arbitrary number of sources, the characteristic polynomial of the equivalent linear system describing the FXLMS algorithm is derived and a method for calculating the stability limit is presented. Also, a related new algorithm free from the above assumption, which is nonlinear with respect to the tap weights, is proposed. Simulation results show that in the early stage of adaptation the new algorithm gives faster decay of errors.
\end{abstract}

Index Terms-Active noise control, convergence analysis, filtered-X LMS (FXLMS) algorithm, multitonal noise.

\section{INTRODUCTION}

A CTIVE noise control (ANC) provides a very useful tool for suppressing low-frequency noises [1], [2]. The primary noise dominated by low frequencies is usually generated by several independent periodic sources such as rotating machines. The principle of ANC is that the control system produces the antinoise field so that the primary sound field from the noise sources is cancelled by destructive interference of sound fields. Typical applications of ANC can be found in transportation systems such as propeller aircrafts, motorboats, helicopters, etc. In such applications, a synchronized signal from each noise source and a multiple-reference control system are usually needed to attain noise reduction efficiently and substantially [3], [4].

A narrow-band multiple-reference feedforward system for ANC with independent noise sources from $K$ rotating machines is shown in Fig. 1. If harmonic components of each noise source are present, we regard such components as other separate noise sources by setting their frequencies as multiples of the fundamental frequency. Each adaptive filter is used to control the amplitude and the phase of a single-frequency reference signal before adding their outputs to drive a secondary source such as a loudspeaker. The microphone is employed to measure the residual noise achieved. The detected error signal is then used to adjust the adaptive filters so as to minimize the level of the residual noise.

Manuscript received March 29, 2004; revised December 3, 2004. The associate editor coordinating the review of this manuscript and approving it for publication was Dr. Shoji Makino.

The authors are with the Department of Systems Science, Graduate School of Informatics, Kyoto University, Kyoto 606-8501, Japan (e-mail: hinamoto@sys.i.kyoto-u.ac.jp; hsakai@ i.kyoto-u.ac.jp).

Digital Object Identifier 10.1109/TSA.2005.854089

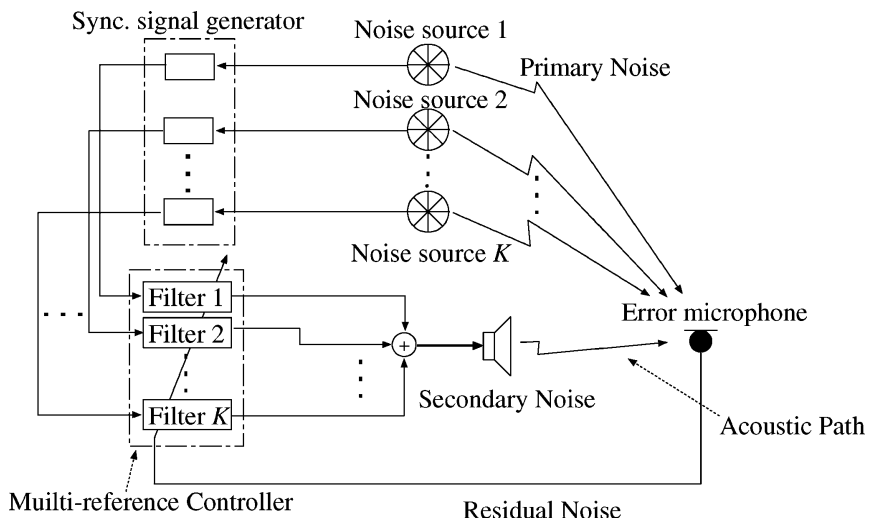

Fig. 1. Physical model of a multireference active noise controller for cancelling multitonal acoustic noise.

Now, with respect to the convergence analysis of such ANC, in the case where a pair of a sinusoid and a cosinusoid is used as reference signals, i.e., in the notch filtering, the analysis of the LMS algorithm using $\mathrm{z}$ transform is well known [5]. Also, a new method using the eigen decomposition of the deterministic correlation matrix has been proposed for the twin-reference case [3]. If the effect of the secondary path is significant, the filtered-X LMS (FXLMS) algorithm is usually employed. In [6], the FXLMS algorithm is employed for the case of one real sinusoid, and the effect of the secondary path to the passband characteristic of the ANC system is analyzed.

In this paper, for the general case of arbitrary number of sources, the analysis of the FXLMS algorithm is performed using a new approach based on a state-space expression. First, the characteristic polynomial of the equivalent linear system describing the FXLMS algorithm is derived. Then, a method for calculating the stability limit about the step size is proposed. Also, by reconsidering the derivation of the FXLMS algorithm under the assumption of slow adaptation limit, a related new algorithm free from this assumption is proposed. So far, in order to be free from the slow adaptation assumption, some alternative algorithms to the FXLMS have been proposed such as a "modified filtered-x" or a "delay compensated filtered-x" algorithm, which utilizes an internal model control structure [7]. However, the structures of the algorithms are rather complicated. The proposed new algorithm has a simple structure using the special property of the input signal. Moreover, it is shown by linearization that our new algorithm is equivalent to the conventional FXLMS algorithm near the convergent point. Therefore, the local stability property is the same as that of the FXLMS algorithm. Finally, we show that the stability limit derived in this 


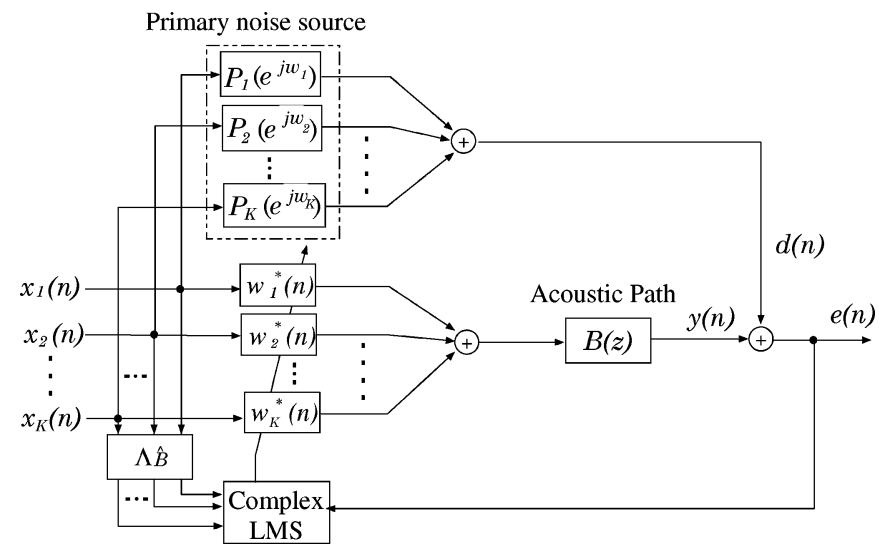

Fig. 2. Block diagram of the whole ANC system for multitonal noise.

paper is in good agreement with the simulation results and the proposed new algorithm gives improved speed of decay of errors in the early stage of adaptation compared with that of the FXLMS algorithm. It is noted that some filtered-x based algorithms with faster convergence have been proposed, based on more sophisticated adaptive filtering methods such as the fast affine projection (FAP), recursive-least-squares (RLS), etc. [1], [8], but, again our new algorithm is much simpler than those algorithms.

The organization of this paper is as follows: Section II presents the derivation of the FXLMS algorithm for active control of multitonal noise and Section III provides its convergence analysis. In Section IV, the proposed new FXLMS algorithm is derived without the assumption of slow adaptation limit and the local stability property of the proposed method near the convergent point is discussed. Section V presents the simulation experiments to show that the stability limits of the conventional and the proposed new FXLMS algorithms coincide well with the corresponding theoretical results.

\section{Active Control of Multitonal Noise WITH THE FXLMS ALGORITHM}

The block diagram of the ANC system is shown in Fig. 2 . An extension to the multichannel case, with multiple actuators and multiple error sensors is straightforward. Here, we treat the single channel case for the sake of brevity. The noise source of the $i$ th rotating machine is assumed to be narrow band and is modeled by a pure complex sinusoid [3]. That is, the $i$ th reference signal to the adaptive system is $x_{i}(n)=e^{j \omega_{i} n}(i=$ $1,2, \ldots, K)$ where $K$ is the total number of noise sources, $\omega_{i}=$ $2 \pi f_{i} / f_{s}, f_{i}$ is the frequency of the $i$ th rotating machine and $f_{s}$ is the sampling rate. When harmonics of one noise source are present, the corresponding multiple pure complex sinusoids are used as the input signals. Let the reference signal vector $\boldsymbol{x}(n)$ and the tap weight vector $\boldsymbol{w}(n)$ be defined respectively as

$$
\begin{aligned}
\boldsymbol{x}(n) & =\left(e^{j \omega_{1} n} e^{j \omega_{2} n} \cdots e^{j \omega_{K} n}\right)^{T} \\
\boldsymbol{w}(n) & =\left[w_{1}(n) w_{2}(n) \cdots w_{K}(n)\right]^{T}
\end{aligned}
$$

where $(\cdot)^{T}$ denotes the transpose and the frequencies $\omega_{i}$ are assumed to be known and distinct. Usually, real-valued systems are treated so that we set a pair of frequency $\omega_{i}$ and $-\omega_{i}$ with complex conjugate pair of initial weights. The transfer function of the secondary path from the loudspeaker to the error microphone is assumed to be an $L$ th order finite impulse response (FIR) system as

$$
B(z)=\sum_{l=0}^{L} b_{l} z^{-l}
$$

This assumption is reasonable, since a physical secondary path of an infinite impulse response (IIR) can be approximated by a FIR system with high order provided that it is stable. The output of the adaptive system is passed through the secondary path to form the antinoise signal $y(n)$ and is expressed in the time domain as

$$
y(n)=b(n) \circledast \boldsymbol{w}^{H}(n) \boldsymbol{x}(n) \equiv \sum_{l=0}^{L} b_{l} \sum_{i=1}^{K} w_{i}^{*}(n-l) x_{i}(n-l)
$$

where $\{b(n)\}$ is the impulse response of the secondary path, and $\circledast,(\cdot)^{H}$ and $(\cdot)^{*}$ denote the convolution operation, the Hermitian transpose and the complex conjugate, respectively. Each tonal signal $x_{i}(n)=e^{j \omega_{i} n}$ is passed through a linear system with the transfer function $P_{i}(z)(i=1,2, \ldots, K)$ so that the desired signal $d(n)$ at the error microphone is written as

$$
d(n)=\sum_{i=1}^{K} P_{i}\left(e^{j \omega_{i}}\right) e^{j \omega_{i} n}=\boldsymbol{w}_{P}^{H} \boldsymbol{x}(n)
$$

where

$$
\boldsymbol{w}_{P}=\left[P_{1}^{*}\left(e^{j \omega_{1}}\right) P_{2}^{*}\left(e^{j \omega_{2}}\right) \cdots P_{K}^{*}\left(e^{j \omega_{K}}\right)\right]^{T} .
$$

If the adaptive tap weights change very slowly, that is, $\boldsymbol{w}(n) \simeq \boldsymbol{w}(n-l)(l=1,2, \ldots, L)$, the antinoise signal (3) is approximated as

$$
y_{s}(n)=\boldsymbol{w}^{H}(n) \Lambda_{B} \boldsymbol{x}(n)
$$

with

$$
\Lambda_{B}=\operatorname{diag}\left[B_{1}, B_{2}, \ldots, B_{K}\right]
$$

where $B_{i}=B\left(e^{j \omega_{i}}\right)(i=1,2, \ldots, K)$. Then, the LMS algorithm for minimizing the mean square of the approximate error signal $e_{s}(n)=d(n)+y_{s}(n)$ is written as

$$
\boldsymbol{w}(n+1)=\boldsymbol{w}(n)-\mu \Lambda_{B} \boldsymbol{x}(n) e_{s}^{*}(n) .
$$

But the actual error is given by $e(n)=d(n)+y(n)$ and sometimes only an approximate knowledge $\hat{B}(z)$ of $B(z)$, which is the transfer function of an $L_{e}$ th order FIR filter, is available. So, the FXLMS algorithm actually used is described by

$$
\boldsymbol{w}(n+1)=\boldsymbol{w}(n)-\mu \Lambda_{\hat{B}} \boldsymbol{x}(n) e^{*}(n)
$$

with

$$
\begin{aligned}
\Lambda_{\hat{B}} & =\operatorname{diag}\left[\hat{B}_{1}, \hat{B}_{2}, \ldots, \hat{B}_{K}\right] \\
\hat{B}_{i} & =\sum_{l=0}^{L_{e}} \hat{b}_{l} e^{-j \omega_{i} l}, \quad(i=1,2, \ldots, K)
\end{aligned}
$$

where $\mu$ is a positive step size. From (3), (4), and (7), the FXLMS algorithm is linear with respect to the adaptive tap weight. Since (7) is derived based on the assumption of slow adaptation limit, there is a room of improvement. 


\section{CONVERgEnCE ANALYSIS OF THE FXLMS AlgORITHM}

By setting $e(n)=0$ with $\boldsymbol{w}(n)=\boldsymbol{w}_{o}$, from (3) and (4), the stationary point of (7) is given by

$$
\boldsymbol{w}_{o}=-\left(\Lambda_{B}^{H}\right)^{-1} \boldsymbol{w}_{P} .
$$

Let the tap error vector $\boldsymbol{v}(n)$ be defined by

$$
\boldsymbol{v}(n)=\boldsymbol{w}(n)-\boldsymbol{w}_{o} .
$$

Then, from (3), (4), and (8), $e(n)$ is simply expressed as

$$
\begin{aligned}
e(n) & =-b(n) \circledast \boldsymbol{w}_{o}^{H} \boldsymbol{x}(n)+b(n) \circledast\left[\boldsymbol{v}^{H}(n)+\boldsymbol{w}_{o}^{H}\right] \boldsymbol{x}(n) \\
& =b(n) \circledast \boldsymbol{v}^{H}(n) \boldsymbol{x}(n) .
\end{aligned}
$$

Substituting (10) into (7), we have

$$
\boldsymbol{v}(n+1)=\boldsymbol{v}(n)-\mu \Lambda_{\hat{B}} \boldsymbol{x}(n)\left\{b(n) \circledast \boldsymbol{v}^{H}(n) \boldsymbol{x}(n)\right\}^{*} .
$$

Writing (11) componentwise for $i=1,2, \ldots, K$, we have

$v_{i}(n+1)=v_{i}(n)-\mu \hat{B}_{i} e^{j \omega_{i} n} \sum_{l=0}^{L} b_{l}^{*} \sum_{k=1}^{K} e^{-j \omega_{k}(n-l)} v_{k}(n-l)$.

The linear system (12) is time-varying but defining $z_{i}(n) \stackrel{\text { def }}{=}$ $e^{-j \omega_{i} n} v_{i}(n)$, then (12) becomes a time-invariant linear system described by

$$
z_{i}(n+1)=e^{-j \omega_{i}} z_{i}(n)-\mu \hat{B}_{i} e^{-j \omega_{i}} \sum_{l=0}^{L} b_{l}^{*} \sum_{k=1}^{K} z_{k}(n-l) .
$$

Defining the vector $\boldsymbol{z}(n)=\left[z_{1}(n) z_{2}(n) \cdots z_{K}(n)\right]^{T},(13)$ is written as

$$
z(n+1)=\Lambda_{\omega}^{*} z(n)-\mu \Lambda_{\hat{B}} \Lambda_{\omega}^{*} \pi \sum_{l=0}^{L} b_{l}^{*} \pi^{H} z(n-l)
$$

where

$$
\Lambda_{\omega}=\operatorname{diag}\left[e^{j \omega_{1}}, e^{j \omega_{2}}, \ldots, e^{j \omega_{K}}\right], \quad \pi=\left(\begin{array}{llll}
1 & 1 & \cdots & 1
\end{array}\right)^{T}
$$

but (14) is expressed as the following state-space form:

$$
\left[\begin{array}{c}
z(n-L+1) \\
z(n-L+2) \\
\vdots \\
z(n+1)
\end{array}\right]=\boldsymbol{G}\left[\begin{array}{c}
z(n-L) \\
z(n-L+1) \\
\vdots \\
z(n)
\end{array}\right]
$$

where the transition matrix $\boldsymbol{G}$ is defined by

$$
\boldsymbol{G}=\left[\begin{array}{cccc}
\mathbf{0} & \boldsymbol{I} & \mathbf{0} & \mathbf{0} \\
\vdots & \ddots & \ddots & \mathbf{0} \\
\mathbf{0} & \cdots & \mathbf{0} & \boldsymbol{I} \\
\mathbf{0} & \cdots & \mathbf{0} & \Lambda_{\omega}^{*}
\end{array}\right]-\mu\left[\begin{array}{c}
\mathbf{0} \\
\vdots \\
\mathbf{0} \\
\Lambda_{\hat{B}} \Lambda_{\omega}^{*} \boldsymbol{\pi}
\end{array}\right]\left(b_{L}^{*} \boldsymbol{\pi}^{H} \cdots b_{0}^{*} \boldsymbol{\pi}^{H}\right)
$$

Thus, the FXLMS algorithm (7) converges to the desired weight vector (8) iff all the eigenvalues $\lambda$ of $G$ are $|\lambda|<1$. To find the

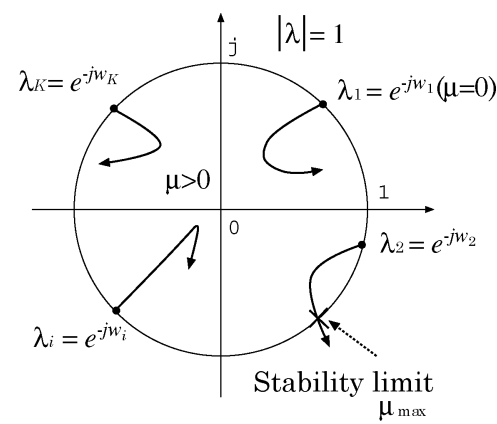

Fig. 3. Movements of the roots of (20) for increasing $\mu$.

eigenvalues, let the eigenvector be $\boldsymbol{c}=\left(\boldsymbol{c}_{0}^{T} \boldsymbol{c}_{1}^{T} \cdots \boldsymbol{c}_{L}^{T}\right)^{T}$, then from $\boldsymbol{G c}=\lambda \boldsymbol{c}$ and (17) we have

$$
\begin{gathered}
\boldsymbol{c}_{1}=\lambda \boldsymbol{c}_{0}, \boldsymbol{c}_{2}=\lambda \boldsymbol{c}_{1}, \ldots, \boldsymbol{c}_{L}=\lambda \boldsymbol{c}_{L-1}, \\
\Lambda_{\omega}^{*} \boldsymbol{c}_{L}-\mu \Lambda_{\hat{B}} \Lambda_{\omega}^{*} \boldsymbol{\pi}\left(b_{L}^{*} \boldsymbol{\pi}^{H} \boldsymbol{c}_{0}+\cdots+b_{0}^{*} \boldsymbol{\pi}^{H} \boldsymbol{c}_{L}\right)=\lambda \boldsymbol{c}_{L} .
\end{gathered}
$$

Hence, from $\boldsymbol{c}_{2}=\lambda^{2} \boldsymbol{c}_{0}, \ldots, \boldsymbol{c}_{L}=\lambda^{L} \boldsymbol{c}_{0}$, we have

$\lambda^{L} \Lambda_{\omega}^{*} \boldsymbol{c}_{0}-\mu \Lambda_{\hat{B}} \Lambda_{\omega}^{*} \boldsymbol{\pi}\left(b_{0}^{*} \boldsymbol{\pi}^{H} \lambda^{L} \boldsymbol{c}_{0}+\cdots+b_{L}^{*} \boldsymbol{\pi}^{H} \boldsymbol{c}_{0}\right)=\lambda^{L+1} \boldsymbol{c}_{0}$.

Since $c_{0} \neq \mathbf{0}$, we have

$$
\left|\lambda^{L+1} \boldsymbol{I}-\lambda^{L} \Lambda_{\omega}^{*}+\mu \Lambda_{\hat{B}} \Lambda_{\omega}^{*} \pi \boldsymbol{\pi}^{H}\left(b_{0}^{*} \lambda^{L}+\cdots+b_{L}^{*}\right)\right|=0 .
$$

Thus, the characteristic equation is given by

$$
\begin{aligned}
\lambda^{L K} & \left(\lambda-e^{-j \omega_{1}}\right) \ldots\left(\lambda-e^{-j \omega_{K}}\right) \\
& \times\left[1+\mu\left(b_{0}^{*} \lambda^{L}+\cdots+b_{L}^{*}\right) \pi^{H} \frac{\left(\lambda \boldsymbol{I}-\Lambda_{\omega}^{*}\right)^{-1}}{\lambda^{L}} \Lambda_{\hat{B}^{\prime}} \Lambda_{\omega}^{*} \pi\right] \\
= & \lambda^{L K}\left(\lambda-e^{-j \omega_{1}}\right) \ldots\left(\lambda-e^{-j \omega_{K}}\right)\left[1+\mu\left(b_{0}^{*}+\cdots+b_{L}^{*} \lambda^{-L}\right)\right. \\
& \left.\times\left(\frac{\hat{B}_{1} e^{-j \omega_{1}}}{\lambda-e^{-j \omega_{1}}}+\cdots+\frac{\hat{B}_{K} e^{-j \omega_{K}}}{\lambda-e^{-j \omega_{K}}}\right)\right]=0 .
\end{aligned}
$$

The roots $\lambda=0$ are stable ones so that they are deleted from (20). Next we obtain the interval of $\mu$ within which the stability condition $|\lambda|<1$ is guaranteed. This process is visualized in Fig. 3. First, for sufficiently small positive $\mu$, let $\lambda=e^{-j \omega_{i}}+a$ where $|a| \ll 1(i=1,2, \ldots, K)$. Substituting this $\lambda$ into (20), we have

$$
\begin{gathered}
a\left(e^{-j \omega_{i}}-e^{-j \omega_{1}}\right) \ldots\left(e^{-j \omega_{i}}-e^{-j \omega_{K}}\right)+\mu\left(b_{0}^{*}+\cdots+b_{L}^{*} e^{j \omega_{i} L}\right) \\
\cdot\left(e^{-j \omega_{i}}-e^{-j \omega_{1}}\right) \ldots\left(e^{-j \omega_{i}}-e^{-j \omega_{K}}\right) \hat{B}_{i} e^{-j \omega_{i}} \simeq 0
\end{gathered}
$$

where $a$ is of order $\mu$ and higher order terms are discarded. Hence, $\lambda \simeq e^{-j \omega_{i}}\left(1-\mu B_{i}^{*} \hat{B}_{i}\right)$. So, for $|\lambda|<1$, it is necessary that the following condition holds:

$$
\operatorname{Re}\left\{B_{i}^{*} \hat{B}_{i}\right\}>0 \quad(i=1,2, \ldots, K) .
$$

This is the well-known $90^{\circ}$ condition in the ANC literature. The corresponding result for the one real sinusoid case is given in $[1$, p. 126]. The stability limit is attained when one eigenvalue 
$\lambda$ first reaches the unit circle, that is, $\lambda=e^{-j \theta}$ as $\mu$ increases where $\theta \neq \omega_{i}(i=1,2, \ldots, K)$. Hence, from (20), we have

$$
1+\mu B_{\theta}^{*}\left[\frac{\hat{B}_{1} e^{-j \omega_{1}}}{e^{-j \theta}-e^{-j \omega_{1}}}+\cdots+\frac{\hat{B}_{K} e^{-j \omega_{K}}}{e^{-j \theta}-e^{-j \omega_{K}}}\right]=0
$$

where $B_{\theta}^{*}=b_{0}^{*}+\cdots+b_{L}^{*} e^{j \theta L}$. Thus, the stability limit $\mu_{\max }$ is obtained by finding the minimum positive value of $\mu_{\theta}$ where

$$
\mu_{\theta}=-\left\{B_{\theta}^{*}\left[\frac{\hat{B}_{1} e^{-j \omega_{1}}}{e^{-j \theta}-e^{-j \omega_{1}}}+\cdots+\frac{\hat{B}_{K} e^{-j \omega_{K}}}{e^{-j \theta}-e^{-j \omega_{K}}}\right]\right\}^{-1}
$$

with $\operatorname{Im}\left\{\mu_{\theta}\right\}=0$. For the special case of $B(z)=1$ with $\hat{B}_{i}=1$ $(i=1,2, \ldots, K)$

$$
\mu_{\theta}=-\left\{\sum_{i=1}^{K} \frac{e^{-j \omega_{i}}}{e^{-j \theta}-e^{-j \omega_{i}}}\right\}^{-1}=\frac{1}{\frac{K}{2}-j \alpha}=\frac{\frac{K}{2}+j \alpha}{\left(\frac{K}{2}\right)^{2}+\alpha^{2}}
$$

where

$$
\alpha=\sum_{i=1}^{K} \frac{\sin \left(\theta-\omega_{i}\right)}{2\left\{1-\cos \left(\theta-\omega_{i}\right)\right\}}=\frac{1}{2} \sum_{i=1}^{K} \cot \frac{\theta-\omega_{i}}{2} .
$$

From $\operatorname{Im}\left\{\mu_{\theta}\right\}=\alpha /\left\{(K / 2)^{2}+\alpha^{2}\right\}=0$, it is readily seen that $\mu_{\max }=2 / K$. This generalizes the result of [3]. For general $B(z)$, we need to compute $\mu_{\max }$ numerically by finding $\mu_{\theta}$ such that $\operatorname{Im}\left\{\mu_{\theta}\right\}=0$ as $\theta$ moves from 0 to $2 \pi$.

\section{Proposed New Algorithm}

Here, we derive a new algorithm free from the assumption of slow adaptation limit. We note, first, that (3) can be rewritten as

$$
\begin{aligned}
y(n)= & w_{1}^{*}(n)\left[\sum_{l=0}^{L} b_{l} \frac{w_{1}^{*}(n-l)}{w_{1}^{*}(n)} e^{-j \omega_{1} l}\right] e^{j \omega_{1} n}+ \\
& \cdots+w_{K}^{*}(n)\left[\sum_{l=0}^{L} b_{l} \frac{w_{K}^{*}(n-l)}{w_{K}^{*}(n)} e^{-j \omega_{K} l}\right] e^{j \omega_{K} n} \\
= & \boldsymbol{w}^{H}(n) \Lambda_{B(n)} \boldsymbol{x}(n)
\end{aligned}
$$

where

$$
\begin{aligned}
\Lambda_{B(n)} & =\operatorname{diag}\left[B_{1}(n), B_{2}(n), \ldots, B_{K}(n)\right] \\
B_{i}(n) & =\sum_{l=0}^{L} b_{l} \frac{w_{i}^{*}(n-l)}{w_{i}^{*}(n)} e^{-j \omega_{i} l} \quad(i=1,2, \ldots, K) .
\end{aligned}
$$

Thus, by replacing $b_{l}(l=0,1, \ldots, L)$ with its estimate $\hat{b}_{l}(l=$ $0,1, \ldots, L_{e}$ ), we obtain the following proposed algorithm:

$$
\boldsymbol{w}(n+1)=\boldsymbol{w}(n)-\mu(n) \Lambda_{\hat{B}(n)} \boldsymbol{x}(n) e^{*}(n)
$$

with a time-varying step size $\mu(n)$ where

$$
\begin{aligned}
e(n) & =d(n)+w^{H}(n) \Lambda_{B(n)} \boldsymbol{x}(n) \\
\Lambda_{\hat{B}(n)} & =\operatorname{diag}\left[\hat{B}_{1}(n), \hat{B}_{2}(n), \ldots, \hat{B}_{K}(n)\right] \\
\hat{B}_{i}(n) & =\sum_{l=0}^{L_{e}} \hat{b}_{l} \frac{w_{i}^{*}(n-l)}{w_{i}^{*}(n)} e^{-j \omega_{i} l} \quad(i=1,2, \ldots, K) .
\end{aligned}
$$

When $w_{i}(n)$ becomes 0 , it is replaced by some small positive number $\epsilon_{1}$ to avoid the division by 0 in (28). From (27) and (28), the proposed algorithm is nonlinear with respect to the adaptive tap weight. Note that we are treating the case where the desired signal which is to be cancelled is a sum of pure sinusoid as in (4); that is, it has line spectrum. Hence, our FXLMS algorithms are not applicable to the case of wide-band stochastic noise. The stationary point of this algorithm is also $\boldsymbol{w}_{o}$ in (8). Also, from (4) and (8) $d^{*}(n)=-\boldsymbol{x}^{H}(n) \Lambda_{B}^{H} \boldsymbol{w}_{o}$. Using the same tap error vector in (9), (27) is rewritten as

$$
\begin{aligned}
\boldsymbol{v}(n+1)= & \boldsymbol{v}(n)-\mu(n) \Lambda_{\hat{B}(n)} \boldsymbol{x}(n)\left[d^{*}(n)+\boldsymbol{x}^{H}(n) \Lambda_{B(n)}^{H} \boldsymbol{w}(n)\right] \\
= & {\left[\boldsymbol{I}-\mu(n) \Lambda_{\hat{B}(n)} \boldsymbol{x}(n) \boldsymbol{x}^{H}(n) \Lambda_{B(n)}^{H}\right] \boldsymbol{v}(n) } \\
& -\mu(n) \Lambda_{\hat{B}(n)} \boldsymbol{x}(n) \boldsymbol{x}^{H}(n)\left[\Lambda_{B(n)}^{H}-\Lambda_{B}^{H}\right] \boldsymbol{w}_{o}
\end{aligned}
$$

but the system (29) is highly nonlinear and is difficult to analyze its convergence property rigorously. So, the local stability property of the proposed FXLMS algorithm near the convergent point is considered here. If the tap error vector becomes small, (29) can be linearized by expanding it with respect to $\boldsymbol{v}(n)$ and $w_{i}(n)(i=1,2, \ldots, K)$ around $\mathbf{0}$ and $w_{o, i}$, which denotes the $i$ th element of $\boldsymbol{w}_{o}$, respectively, with a fixed step size $\mu$. That is, denoting the $i$ th diagonal element $\Lambda_{B(n)}-\Lambda_{B}$ by $\left[\Lambda_{B(n)}-\Lambda_{B}\right]$, we have

$$
\begin{aligned}
& {\left[\Lambda_{B(n)}-\Lambda_{B}\right]_{i}} \\
& \quad=\sum_{l=0}^{L} b_{l} \frac{v_{i}^{*}(n-l)-v_{i}^{*}(n)}{w_{i}^{*}(n)-w_{o, i}^{*}+w_{o, i}^{*}} e^{-j \omega_{i} l} \\
& \simeq \sum_{l=0}^{L} b_{l} \frac{v_{i}^{*}(n-l)-v_{i}^{*}(n)}{w_{o, i}^{*}}\left(1-\frac{w_{i}^{*}(n)-w_{o, i}^{*}}{w_{o, i}^{*}}\right) e^{-j \omega_{i} l} \\
& \simeq \sum_{l=0}^{L} b_{l} \frac{v_{i}^{*}(n-l)-v_{i}^{*}(n)}{w_{o, i}^{*}} e^{-j \omega_{i} l}
\end{aligned}
$$

where higher order errors concerning $v_{i}(n)$ and $w_{i}^{*}(n)-w_{o, i}^{*}$ are discarded. From (29) and (30), the tap error vector $\boldsymbol{v}(n+1)$ can be approximated as

$$
\begin{aligned}
\boldsymbol{v}(n+1) \simeq & \boldsymbol{v}(n)-\mu \Lambda_{\hat{B}(n)} \boldsymbol{x}(n) \boldsymbol{x}^{H}(n) \Lambda_{B(n)}^{H} \boldsymbol{v}(n) \\
& +\mu \Lambda_{\hat{B}(n)} \boldsymbol{x}(n) \boldsymbol{x}^{H}(n) \\
& \times\left[\begin{array}{lll}
\ddots & & 0 \\
& \sum_{l=0}^{L} b_{l}^{*} \frac{v_{i}(n)-v_{i}(n-l)}{w_{o, i}} e^{j \omega_{i} l} & \\
\mathbf{0} & \ddots
\end{array}\right]\left[\begin{array}{c}
\vdots \\
w_{o, i} \\
\vdots
\end{array}\right] \\
\simeq & \boldsymbol{v}(n)-\mu \Lambda_{\hat{B}} \boldsymbol{x}(n) \boldsymbol{x}^{H}(n) \Lambda_{B}^{H} \boldsymbol{v}(n) \\
& +\mu \Lambda_{\hat{B}} \boldsymbol{x}(n)\left[\boldsymbol{x}^{H}(n) \Lambda_{B}^{H} \boldsymbol{v}(n)-\left\{b(n) \circledast \boldsymbol{v}^{H}(n) \boldsymbol{x}(n)\right\}^{*}\right] \\
= & \boldsymbol{v}(n)-\mu \Lambda_{\hat{B}} \boldsymbol{x}(n)\left\{b(n) \circledast \boldsymbol{v}^{H}(n) \boldsymbol{x}(n)\right\}^{*} .
\end{aligned}
$$

This shows that from (11) near the stationary point $\boldsymbol{w}_{o}$ the behavior of the proposed new FXLMS algorithm is almost the same as that of the FXLMS algorithm, provided its trajectory approaches to this region. Thus, the stability limit of the FXLMS algorithm gives some insight to that of the proposed one.

Now, we consider the case where $\hat{B}(z)=B(z)$ and the step size $\mu=\mu(n)$ is time varying. It is seen that the second term 
in the right-hand side of (29) is $O\left(\mu^{2}\right)$, since $\Lambda_{B(n)}-\Lambda_{B}=$ $O(\mu)$. Hence, for $\mu \ll 1$, this term can be discarded. Defining $\boldsymbol{p}(n) \stackrel{\text { def }}{=} \Lambda_{B(n)} \boldsymbol{x}(n)$, we have

$$
\boldsymbol{v}(n+1) \simeq\left[\boldsymbol{I}-\mu(n) \boldsymbol{p}(n) \boldsymbol{p}^{H}(n)\right] \boldsymbol{v}(n) .
$$

Then, from (32), the squared norm of $\boldsymbol{v}(n+1)$ is given by

$$
\begin{aligned}
\|\boldsymbol{v}(n+1)\|^{2} & \simeq \boldsymbol{v}^{H}(n)\left[\boldsymbol{I}-\mu(n) \boldsymbol{p}(n) \boldsymbol{p}^{H}(n)\right]^{2} \boldsymbol{v}(n) \\
& =\|\boldsymbol{v}(n)\|^{2}-\mu(n)\left[2-\boldsymbol{p}^{H}(n) \boldsymbol{p}(n) \mu(n)\right]\left|\boldsymbol{v}^{H}(n) \boldsymbol{p}(n)\right|^{2} .
\end{aligned}
$$

Since the difference between $\|\boldsymbol{v}(n)\|^{2}$ and $\|\boldsymbol{v}(n+1)\|^{2}$ is written as

$$
\begin{aligned}
\|\boldsymbol{v}(n+1)\|^{2}-\|\boldsymbol{v}(n)\|^{2} \\
\\
\simeq-\mu(n)\left[2-\boldsymbol{p}^{H}(n) \boldsymbol{p}(n) \mu(n)\right]\left|\boldsymbol{v}^{H}(n) \boldsymbol{p}(n)\right|^{2}
\end{aligned}
$$

$\|\boldsymbol{v}(n)\|$ is nonincreasing for $0<\eta<2$ where

$$
\mu(n)=\frac{\eta}{\boldsymbol{p}^{H}(n) \boldsymbol{p}(n)}=\frac{\eta}{\left\|\Lambda_{B(n)} \boldsymbol{x}(n)\right\|^{2}}
$$

but the upper limit 2 is invalid, since we assume that $\mu$ is small and the second term of the right-hand side of (29) is discarded. If the trajectory of (27) with (35) approaches the stationary point $\boldsymbol{w}_{o}, \mu(n)$ can be written as

$$
\begin{aligned}
\mu(n) & =\frac{\eta}{\left|B_{1}(n)\right|^{2}+\left|B_{2}(n)\right|^{2}+\cdots+\left|B_{K}(n)\right|^{2}} \\
& \simeq \frac{\eta}{\left|B_{1}\right|^{2}+\left|B_{2}\right|^{2}+\cdots+\left|B_{K}\right|^{2}} .
\end{aligned}
$$

Hence, using the upper limit $\mu_{\max }$ in (24) for the FXLMS algorithm, the upper limit $\eta_{\max }$ for the proposed FXLMS algorithm is given by

$$
\eta_{\max } \simeq\left(\left|B_{1}\right|^{2}+\left|B_{2}\right|^{2}+\cdots+\left|B_{K}\right|^{2}\right) \mu_{\max } .
$$

For the case of $\hat{B}(z) \neq B(z)$, we use

$$
\mu(n)=\frac{\eta}{\left\|\Lambda_{\hat{B}(n)} x(n)\right\|^{2}}
$$

in (27) with the upper limit of $\eta$ given by

$$
\eta_{\max }=\left(\left|\hat{B}_{1}\right|^{2}+\left|\hat{B}_{2}\right|^{2}+\cdots+\left|\hat{B}_{K}\right|^{2}\right) \mu_{\max } .
$$

Table I shows the numerical complexities (operations per sample) of the FXLMS algorithm and the proposed algorithm where $K$ and $L_{e}$ refer to the total number of noise sources and the order of the estimated secondary path, respectively. It is seen that the proposed algorithm requires much computations for large $L_{e}$.

\section{Simulation Results}

To examine the validity of the above theoretical developments, some simulations have been performed. Here, we treat the following two cases.
TABLE I

COMPLEXITY (OPERATIONS PER SAMPLE) OF THE FXLMS AND THE PROPOSED ALGORITHM

\begin{tabular}{lrr}
\hline & FXLMS & proposed method \\
\hline Multiplications & $3 K+1$ & $\left(L_{e}+5\right) K+1$ \\
Divisions & 0 & $K+1$ \\
Additions & $2 K-1$ & $\left(L_{e}+3\right) K-2$ \\
\hline
\end{tabular}

( $K$ and $L_{e}$ are the total number of noise sources and the order of the estimated secondary path, respectively.)

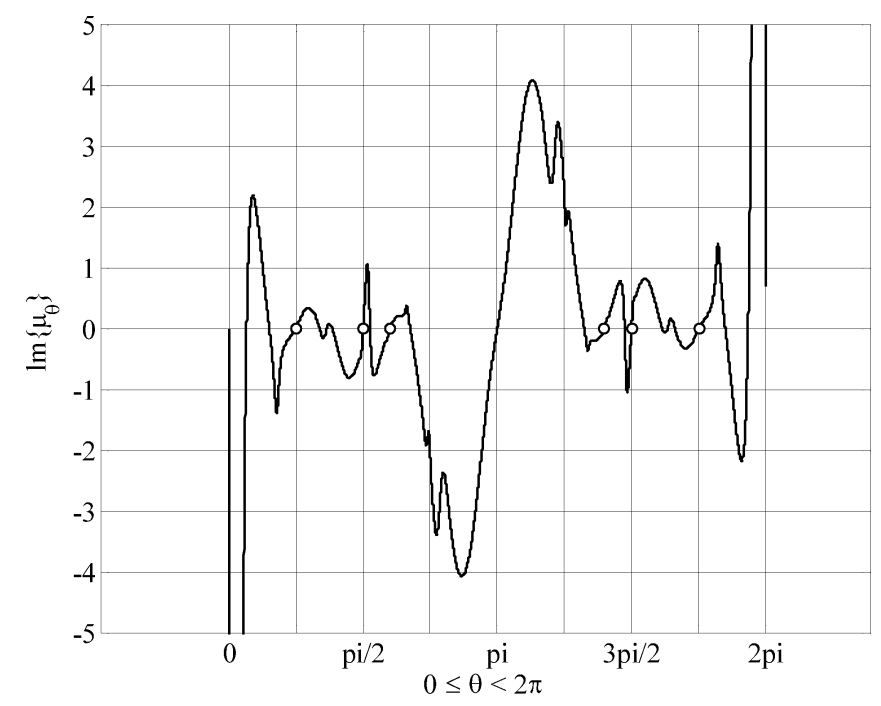

Fig. 4. Graph of $\operatorname{Im}\left\{\mu_{\theta}\right\}$ in (24) versus $\theta$ for Case 1 .

Case 1: $B(z)=\hat{B}(z)$ where $B(z)$ is the transfer function of a 256 tap FIR system $\left(L=L_{e}=256\right)$ which corresponds to a real secondary path in [1].

Case 2: $B(z)$ is the same as Case $1(L=256) . \hat{B}(z)$ is a 32 tap FIR system by retaining the first 32 taps of $B(z)$ $\left(L_{e}=32\right)$.

The secondary path $B(z)$ is represented by an IIR system whose numerator and denominator coefficients are in the files s_z.bin and s_p.bin in [1], respectively. In the simulations, we used a FIR system with 256 taps, which is obtained by truncating the power-series expansion of $B(z)$ and scaling by dividing by three times the maximum absolute value of its impulse response coefficients. $B(z)$ and $\hat{B}(z)$ in Case 2 satisfy the $90^{\circ}$ condition in (22).

The reference signals for the FXLMS algorithm are assumed to be of the form of unit magnitude complex sinusoid, but to treat real-valued systems, we set a pair of frequency $\omega_{i}$ and $-\omega_{i}$ with the same step size and complex conjugate pair of initial weights. In our simulation studies, we consider three tonal noise frequencies of 125, 250 (harmonic of $125 \mathrm{~Hz}$ ), and $300 \mathrm{~Hz}$ and a sampling frequency of $1 \mathrm{kHz}$, which gives rise to $\omega_{1}=\pi / 4$, $\omega_{2}=\pi / 2$ and $\omega_{3}=3 \pi / 5$. So, we have $K=6$ with $\boldsymbol{x}(n)=$ $\left(e^{j \pi n / 4} e^{-j \pi n / 4} e^{j \pi n / 2} e^{-j \pi n / 2} e^{j 3 \pi n / 5} e^{-j 3 \pi n / 5}\right)^{T}$ and each 


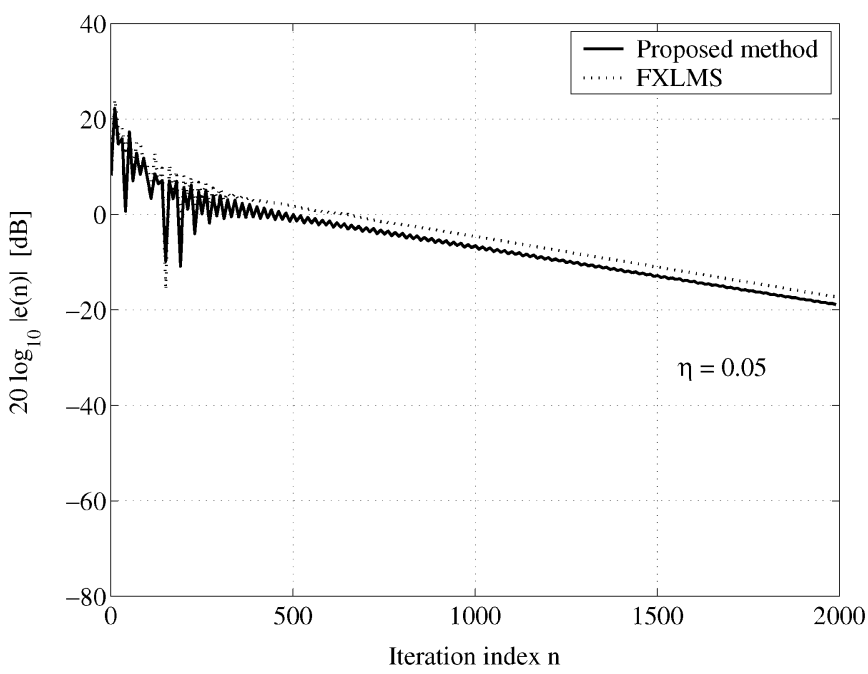

(a)

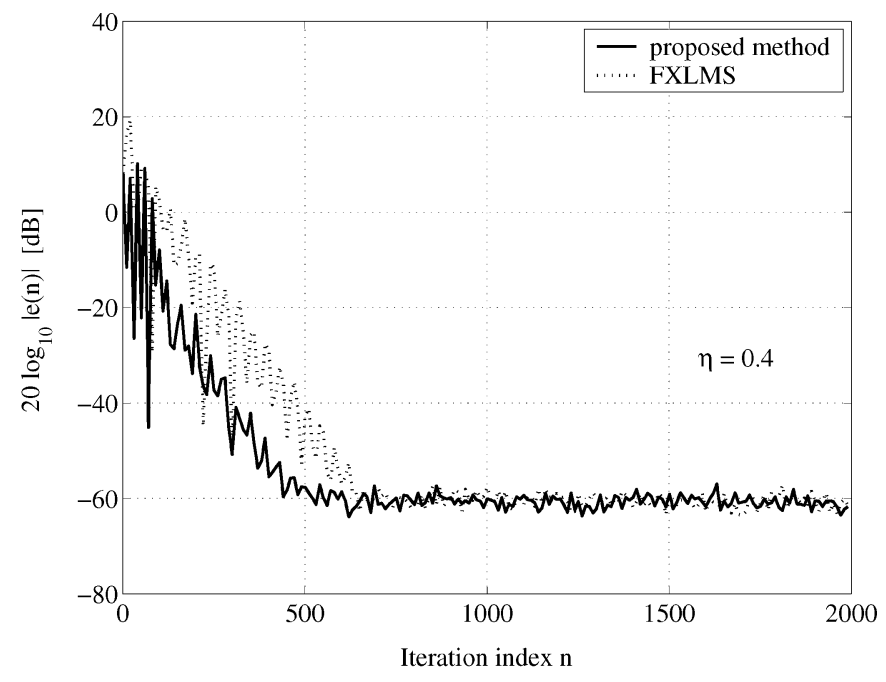

(b)

Fig. 5. Learning curves in decibels for Case $1\left(L_{e}=256\right.$ ) with $\eta=0.05$ and $\eta=0.4$. (a) $\eta=0.05$. (b) $\eta=0.4$.



(a)

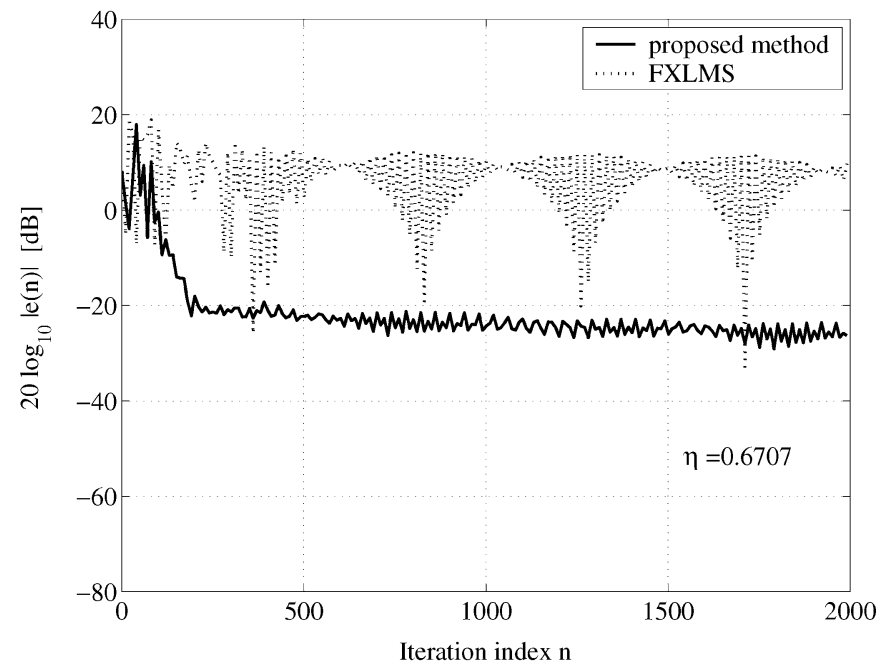

(b)

Fig. 6. Learning curves in decibels for Case $1\left(L_{e}=256\right.$ ) with $\eta=0.5$ and $\eta_{\max }=0.6707$. (a) $\eta=0.5$. (b) $\eta_{\max }=0.6707$.

tonal signal $x_{i}(n)=e^{j \omega_{i} n}$ is passed through either of two primary paths $P_{1}(z)$ or $P_{2}(z)$ which are taken from [1] where the files p1z.bin and p2z.bin contain the numerator coefficients of $P_{1}(z)$ and $P_{2}(z)$, respectively, and p1p.bin and p2p.bin contain the denominator coefficients. $P_{1}(z)$ and $P_{2}(z)$ were scaled similary as for $B(z)$. Hence, the transfer function vector from the primary source to the microphone

$$
\begin{aligned}
\boldsymbol{w}_{P}=\left[P_{1}\left(e^{j \pi / 4}\right),\right. & P_{1}\left(e^{-j \pi / 4}\right), P_{1}\left(e^{j \pi / 2}\right), \\
& \left.P_{1}\left(e^{-j \pi / 2}\right), P_{2}\left(e^{j 3 \pi / 5}\right), P_{2}\left(e^{-j 3 \pi / 5}\right)\right]^{H} .
\end{aligned}
$$

From (8), the stationary (optimal) weight $\boldsymbol{w}_{o}$ can be obtained and it turns out that the absolute value of $w_{o, i}(i=1, \ldots, K)$ is nearly 1 .

First, we obtain the upper limit $\mu_{\max }$ in (24) numerically. In Fig. 4, for Case 1, the graph of $\operatorname{Im}\left\{\mu_{\theta}\right\}$ is presented. Besides at $\theta=\pi / 4, \pi / 2,3 \pi / 5,7 \pi / 5,3 \pi / 2,7 \pi / 4$, which are excluded, $\operatorname{Im}\left\{\mu_{\theta}\right\}$ becomes 0 at 16 points. Among these points, the minimum value of $\mu$ is found to be 0.5291 . Hence, in this case, we have $\mu_{\max }=0.5291$. Since the proposed algorithm is normalized as in (35), from (37), the upper limit of the proposed algorithm is $\eta_{\max }=\left\|\Lambda_{B} \boldsymbol{x}(n)\right\|^{2} \mu_{\max }=0.6707$. Similarly, for Case 2, the upper limit is found to be $\mu_{\max }=0.5167$ and $\eta_{\max }=\left\|\Lambda_{\hat{B}} \boldsymbol{x}(n)\right\|^{2} \mu_{\max }=0.7321$.

Next, we compare the convergence characteristics of the FXLMS and the proposed algorithm. For the FXLMS algorithm, $\mu$ is set to $\eta /\left(\left|B_{1}\right|^{2}+\cdots+\left|B_{K}\right|^{2}\right)$ for Case 1 and $\eta /\left(\left|\hat{B}_{1}\right|^{2}+\cdots+\left|\hat{B}_{K}\right|^{2}\right)$ for Case 2. For the proposed algorithm, the same $\eta$ is used in (35) and (38). To see the performance floor easily, weak white noise is added to $d(n)$ so that the error level is $-60 \mathrm{~dB}$. The performance is obtained by the average of 20 trials. Since usually we do not have a priori knowledge about $\boldsymbol{w}_{o}$, here we use a relatively large initial weight $w_{i}(0)=10$ $(i=1, \ldots, K)$.

Figs. 5 and 6 show the plots of the magnitude of the error $e(n)$ in decibels for Case 1 with $\eta=0.05,0.4,0.5$, and 0.6707, respectively. From these figures, when $\eta$ is relatively small (for example $\eta=0.05$ ), the performance of the proposed algorithm 


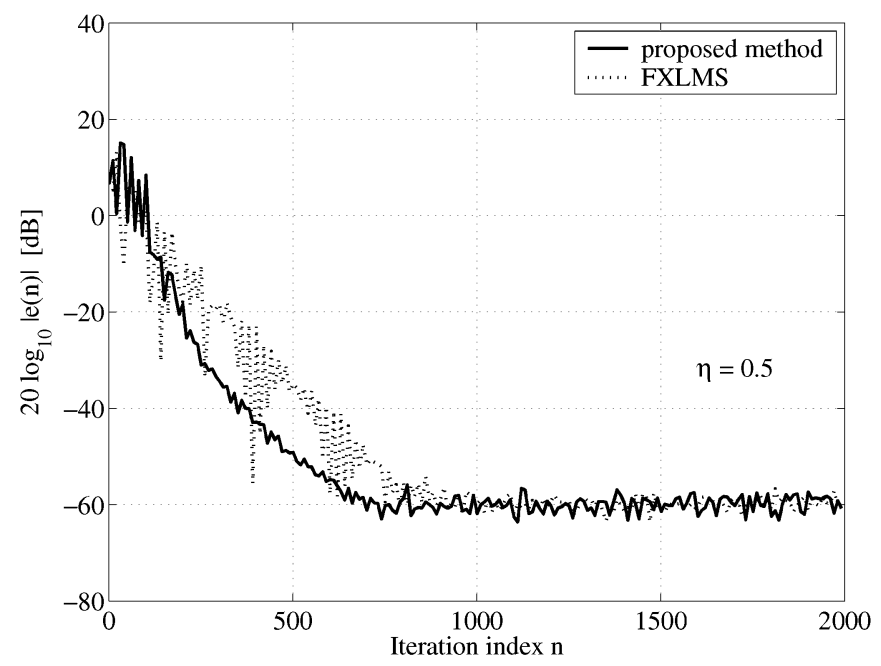

(a)



(b)

Fig. 7. Learning curves in decibels for Case $1\left(L_{e}=256\right)$ using different initial weights. (a) $w_{i}(0)=5(i=1, \ldots, K)$ for $\eta=0.5$. (b) $w_{i}(0)=100$ $(i=1, \ldots, K)$ for $\eta=0.5$.

is a little better than that of the FXLMS algorithm and both algorithms converge slowly, but the choice of $\eta=0.4$ gives the fastest convergence speed for the FXLMS algorithm and the proposed algorithm has a faster convergence than the FXLMS algorithm. For $\eta=0.5$, the FXLMS algorithm slows down but the proposed algorithm converges as fast as the FXLMS for $\eta=0.4$. We see that for $\eta<\eta_{\max }=0.6707$, both algorithms always converge to $-60 \mathrm{~dB}$ and in Fig. 6 (b) for the stability limit $\eta_{\max }$, still the error of the proposed algorithm decays to a certain level whereas the FXLMS algorithm does not converge at all. It is also observed that, for $\eta_{\max }<\eta$, the FXLMS algorithm diverges, but the proposed algorithm converges to a certain level above $-60 \mathrm{~dB}$, and for $2 \leq \eta$, the proposed algorithm diverges. Thus, the proposed algorithm has some robustness concerning the choice of the step size. Also, in Case 2, we have confirmed that almost the same tendency of the performance as Case 1 is observed.

Fig. 7 shows the plots of the magnitude of the error $e(n)$ in decibels for Case 1 with $\eta=0.5$ using two initial weights $w_{i}(0)=5$ and $100(i=1, \ldots, K)$ in order to investigate the effect of the initial weight. From Fig. 7, with $w_{i}(0)=5$ $(i=1, \ldots, K)$, the difference of the performance of both algorithms becomes small but with $w_{i}(0)=100(i=1, \ldots, K)$, the difference becomes large. It is seen that the proposed algorithm can eliminate the effect of large initial weight errors. For the FXLMS algorithm, the tap error vector obeys a linear time-invariant (16) so that the decay of the magnitude of error in decibels is linear. This can be seen from these learning curves. For the proposed algorithm, after the tap error becomes small, the corresponding decay is also linear, but in the early stage of adaptation, the decay is much faster.

Finally, Fig. 8 plots the change of the following quantity:

$$
\gamma(n)=\sum_{i=1}^{K} \sum_{l=1}^{L_{e}}\left|\hat{b}_{l} \frac{w_{i}(n)-w_{i}(n-l)}{w_{i}(n)}\right|
$$

in decibels for the setting in Fig. 6(a). This is a sum of the upper bound of $\left|\hat{B}_{i}-\hat{B}_{i}(n)\right|$ and is related in some sense to the vari-

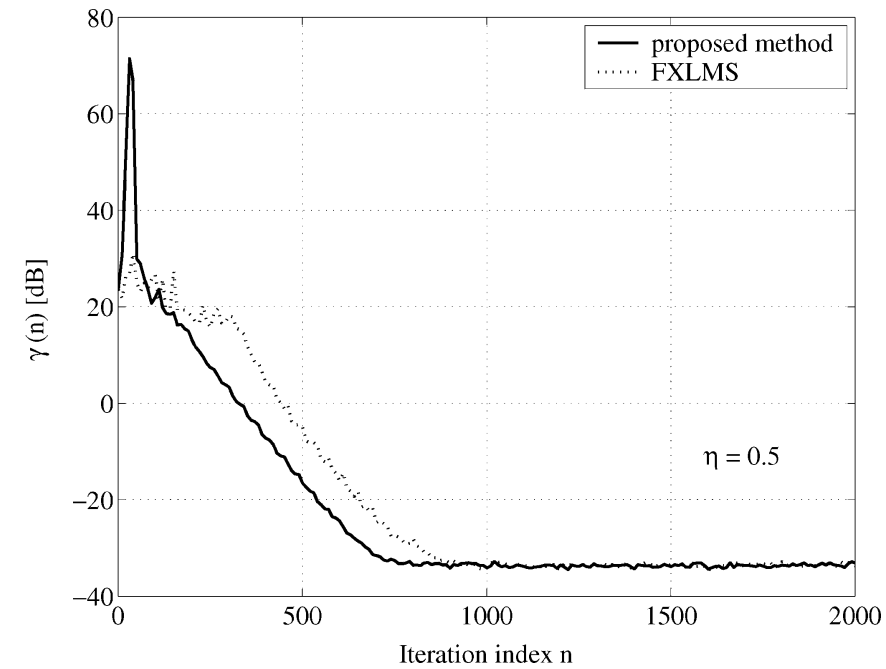

Fig. 8. Plots of $\gamma(n)$ in decibels from (40) for the setting in Fig. 6(a) $\left(L_{e}=\right.$ $256)$.

ation of the adaptive tap weights. From Figs. 6(a) and 8, if $\gamma(n)$ is large, that is, the variation of the adaptive filter is large, the difference between the performance of both algorithms is large. In other words, when the assumption of slow adaptation limit is not realized, it can be concluded that the proposed method is effective.

\section{CONCLUSION}

In this paper, first we have presented analysis of the FXLMS algorithm with tonal reference signals. The characteristic equation is derived and a procedure for computing the stability limit is given. Then, a related new FXLMS algorithm has been proposed. By simulations, it is found that the latter algorithm gives faster decay of errors in the early stage and has some robustness about the choice of the step size. It is a future work to examine these properties in detail. 


\section{REFERENCES}

[1] S. M. Kuo and D. R. Morgan, Active Noise Control Systems. New York: Wiley, 1996.

[2] C. R. Fuller, S. J. Elliot, and P. A. Nelson, Active Control of Vibrations. New York: Academic, 1996

[3] S. Johansson, S. Nordebo, and I. Claesson, "Convergence analysis of a twin-reference complex least-mean-squares algorithm," IEEE Trans. Speech Audio Process., vol. 10, no. 3, pp. 213-221, May 2002.

[4] S. Johansson, I. Claesson, S. Nordebo, and P. Sjösten, "Evaluation of multiple reference active noise control algorithms on Dornier 328 aircraft data," IEEE Trans. Speech Audio Process., vol. 7, pp. 473-477, Jul. 1999.

[5] B. Widrow and S. D. Stearns, Adaptive Signal Processing. Upper Saddle River, NJ: Prentice-Hall, 1985.

[6] S. M. Kuo and M. Ji, "Passband disturbance reduction in periodic active noise control system," IEEE Trans. Speech Audio Process., vol. 4, no. 2, pp. 96-103, Mar. 1996.

[7] M. Bouchard and S. Norcross, "Computational load reduction of fast convergence algorithms for multichannel active noise control," Signal Process., vol. 83, no. 1, pp. 121-134, Jan. 2003.

[8] M. Bouchard, "Multichannel fast affine projection algorithm for active noise control," in Proc. Int. Conf. Acoustics, Speech, and Signal Processing, vol. 2, Orlando, FL, May 2002, pp. 1873-1876.

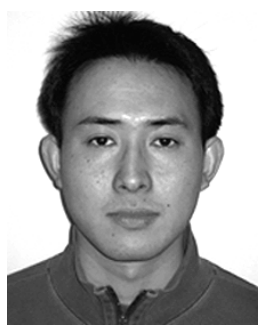

Yoichi Hinamoto (S'04) was born in Kobe, Japan, in 1979. He received the B.E. degree in electrical and electronic engineering from University of Tokushima, Japan, in 2001 and the M.E. degree in information science from the Nara Institute of Science and Technology (NAIST), Japan, in 2003. $\mathrm{He}$ is currently pursuing the Ph.D. degree at the Graduate School of Informatics, Kyoto University, Kyoto, Japan.

His research interests include adaptive signal processing and active noise control.

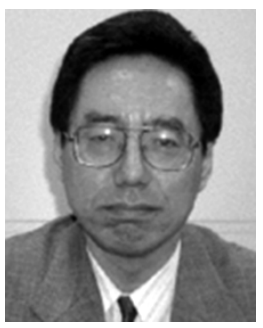

Hideaki Sakai (M'78-SM'02) received the B.E. and Dr.Eng. degrees in applied mathematics and physics, respectively, from Kyoto University, Kyoto, Japan, in 1972 and 1981, respectively.

From 1975 to 1978 , he was with Tokushima University, Japan. From 1987 to 1988, he spent six months at Stanford University, Stanford, CA, as a Visiting Scholar. He was an Associate Editor of the IEICE Transactions Fundamentals of Electronics, Communications, and Computer Sciences from 1996 to 2000 and is currently on the editorial board of the EURASIP Journal of Applied Signal Processing. He is currently a Professor in the Department of Systems Science, Graduate School of Informatics, Kyoto University. His research interests are in the area of statistical and adaptive signal processing.

Dr. Sakai was an Associate Editor of IEEE TRANSACTIONS ON SIGNAL PROCESSING from 1999 to 2001. 